\title{
Auswirkungen des Pilotversuchs BDA auf die Grundwasserverhältnisse
}

\author{
Julia Derx · Alfred Paul Blaschke
}

Online publiziert: 24. März 2016

(C) Die Autor(en) 2016. Dieser Artikel ist auf Springerlink.com mit Open Access verfügbar.

Zusammenfassung In dieser Arbeit wurde untersucht, welchen Einfluss die Summe der baulichen Maßnahmen im Bereich der Pilotversuchsstrecke Bad Deutsch Altenburg auf die Grundwasserverhältnisse haben. Als methodische Vorgangsweise wurde eine Vergleichsdarstellung der Bedingungen der Austauschprozesse zwischen Grund- und Flusswasser vor und nach den Baumaßnahmen gewählt. Die bisherigen Analysen der Grundwasserverhältnisse vor und nach den Baumaßnahmen im Pilotversuch BDA zeigen keine wesentliche Beeinflussung der Grundwasserverhältnisse. Dies ist weitgehend auf die effluenten Verhältnisse zwischen Grundund Oberflächenwasser in diesem Streckenabschnitt der Donau zurückzuführen. Eine Übertragung dieser Ergebnisse auf andere Streckenabschnitte der Donau mit anderen Rahmenbedingungen ist nur bedingt möglich. Es zeigt sich, dass eine Aufrechterhaltung der Interaktion des Grundwassers mit der Donau und deren Seitenarme eine wesentliche Bedeutung für die Erhaltung der Grundwasserverhältnisse haben. Deshalb sind zur Erhaltung der Grundwasserverhältnisse weitere Maßnahmen gegen ein Absinken der Oberflächenwasserstände aus wasserwirtschaftlicher Sicht erforderlich.

DI Dr. J. Derx $(\varangle) \cdot$ ao.Univ.-Prof. DI Dr. A. P. Blaschke

Institut für Wasserbau und

Ingenieurhydrologie, Interuniversity Cooperation Centre Water and Health (ICC Water \& Health, www.waterandhealth. at), Centre for Water Resource Systems, TU Wien,

Karlsplatz 13,

1040 Wien, Österreich

E-Mail: derx@hydro.tuwien.ac.at

ao.Univ.-Prof. DI Dr. A. P. Blaschke

E-Mail: blaschke@hydro.tuwien.ac.at
Impact of the BDA pilot test on groundwater conditions

Abstract This study investigates the impact of the sum of the structural measures on groundwater conditions in the context of the pilot test stretch in Bad Deutsch-Altenburg (BDA). The methodological approach was a comparison of the conditions for the exchange processes between groundwater and river water pre and post the selected structural work. The analyses of the groundwater conditions pre and post the structural work in the BDA pilot test show no substantial impact on the groundwater conditions so far. This is largely due to the effluent conditions between groundwater and surface water along this stretch of the Danube. The transfer of these results to other sections of the Danube with other boundary conditions is only partly possible. It appears that the continuous interaction of groundwater with the waters of the Danube and its branches is of significant importance for the conservation of the groundwater conditions. Therefore, from a water-management point of view, further measures against a decline in surface-water levels are essential for conserving the groundwater conditions.

\section{Einleitung}

Das Ziel dieser Arbeit ist es, die kausalen Zusammenhänge zwischen den Eingriffen im Bereich der Pilotversuchsstrecke und den wasserwirtschaftlichen Grundwasserverhältnissen $\mathrm{zu}$ beschreiben und aufzuzeigen. Dabei werden die Veränderungen untersucht, die durch die im Abschnitt der Donau von Strom-km 1887,5 bis Strom-km 1884,5 ausgeführten baulichen Maßnahmen bedingt sind, wie granulometrische Sohlverbesserung (GSV), Niederwasserregulierung mit Bau, Umbau und Rückbau von Buhnen, Stromsohlenanpassung, Uferrückbau und Uferabsenkung und eine verbesserte Anbindung von Seitenarmen. Die baulichen Maßnahmen bewirken, zusätzlich $\mathrm{zu}$ natürlichen Veränderungen in der Donau, eine Änderung der Morphologie der Donausohle und der Uferzonen. Dies kann auch zu geänderten Bedingungen für die Austauschprozesse zwischen Grund- und Flusswasser führen. $\mathrm{Ob}$ und wie sich dies im Bereich der Pilotversuchsstrecke verändert, wurde und wird mithilfe von Grundwasserstandsmessungen, Untersuchungen der Grundwasserqualität, Untersuchungen der Donausohle mithilfe von Freeze-Cores und -Panels sowie visuell (Fotos der Donausohle in einem dichten Raster) und durch Bestimmungen der Sohldurchlässigkeit im Johler Arm mithilfe von Versickerungsversuchen erfasst. Zur Veranschaulichung werden Ergebnisse der Auswertungen mittels Vergleichsdarstellungen der Grundwasserverhältnisse zwischen dem Ist-Zustand vor und nach der Umsetzung der baulichen Maßnahmen dargestellt und diskutiert.

Insbesondere werden die folgenden Fragen untersucht: Ändern sich die Grundwasserspiegellagen durch die Summe aller durchgeführten baulichen Maßnahmen? Kommt es zu Auswirkungen auf den Austausch von Interstitialwasser in Schotterkörpern? Ist eine Veränderungen der Korngrößenverteilung der obersten Steinlage durch die GSV feststellbar? Welche Sedimente lagern sich in den Buhnenfeldern ab? Ändert sich die Durchlässigkeit der Gewässersohle im Böschungsbereich bzw. im Johler Arm? Ändert sich die Qualität des Grundwassers? Kommt es zu Veränderungen im Bereich der Thermalwasserquellen in Bad DeutschAltenburg?

Die hier getroffenen Aussagen beziehen sich auf Auswertungen der vorhandenen Daten für manche Fragestellungen (z. B. Veränderung der Donausohle) für noch relativ kurze Zeit- 


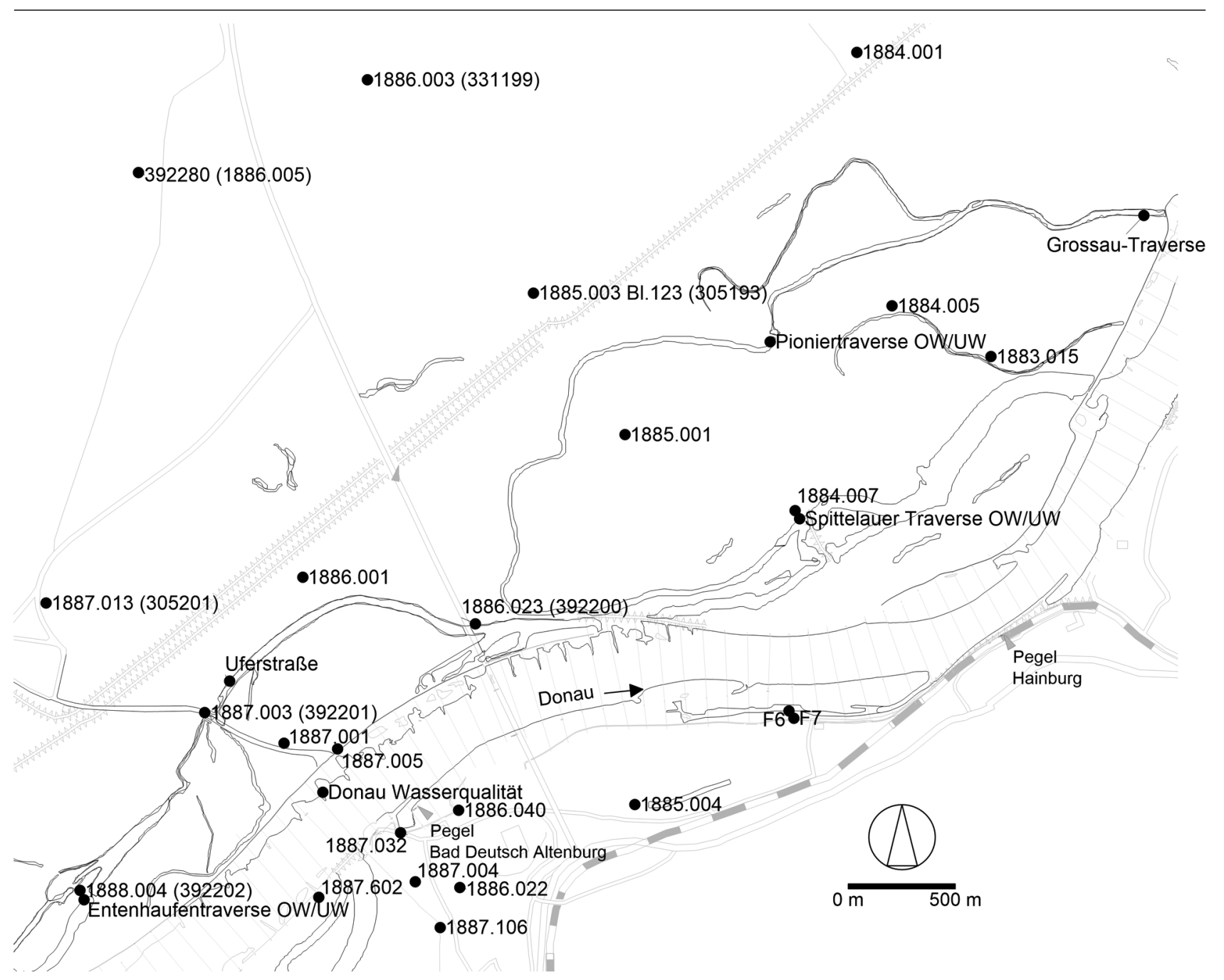

Abb. 1 Lage der Grundwasser-Messstellen und Oberflächen-Pegel

räume nach Fertigstellung. Im Zuge des noch über Jahre weiter zu führenden Monitoringprogramms sind daher auch Aussagen über die Aussagekraft der Messparameter zu erwarten. Zusätzlich sollen die abiotischen und biotischen Aspekte verstärkt in Zusammenhang gebracht werden und damit weitere Grundlagen für die Analyse der großräumigen Auswirkungen schaffen.

\section{Methoden}

Diese Arbeit basiert einerseits auf den Zwischenauswertungen der Daten aus der Zeit vor Baudurchführung (Blaschke und Derx 2009) und auf den Auswertungen der Messungen nach Baudurchführung (Derx und Blaschke 2015). Um unterschiedliche hydrologische Situationen miteinbeziehen zu können, sind die vorgesehenen längeren Beobachtungen erforderlich, und daher sind für die meisten angeführten Fragestellungen die hier gezeigten Ergebnisse als vorläufig anzusehen.

Die Messungen von Grundwasserstand, Temperatur, Leitfähigkeit und Grundwasserqualität wurden gemäß den Vorgaben im Wasserrechtsbescheid durchgeführt und durch zusätzliche Messungen, wie Freeze-Cores und -Panels, Durchlässigkeitsversuche und Visualisierung der Donausohle im Rahmen des Monitoringprogramms ergänzt. Die gewählten Zeitabschnitte vor und nach Baudurchführung wurden hier mit Jänner 2006 bis September 2007 bzw. von März 2009 bis Dezember 2015 festgesetzt.

\subsection{Untersuchung der Grundwasserstände}

In Abb. 1 ist die Lage der Grundwassermessstellen, der Donaupegel und der
Probenahmestellen für die Untersuchungen der Wasserqualität im Bereich des Pilotversuchs Bad Deutsch-Altenburg dargestellt. Um den zeitlichen Trend der Grundwasserstände zu untersuchen, wurde eine lineare Trendanalyse für den Zeitabschnitt jeweils vor und nach den Baumaßnahmen durchgeführt. Um die Grundwasserströmungsrichtungen $\mathrm{zu}$ untersuchen, eignet sich die Darstellung der Grundwasserisolinien. Diese Darstellung wurde jeweils für einen Zeitpunkt vor und nach der Baudurchführung erstellt, an denen eine mittlere Donauwasserführung vorherrschte. Da die Grundwassersituation im Hinterland von den jahreszeitlich schwankenden hydrologischen Bedingungen abhängt, wurde bei der Auswahl der Zeitpunkte auf ähnliche Grundwasserstände geachtet. Als Eingangsdaten dienten kontinuierliche Messdaten auf Stundenbasis sowie Tageswerte, welche - basierend auf wöchent- 


\section{Originalarbeit}

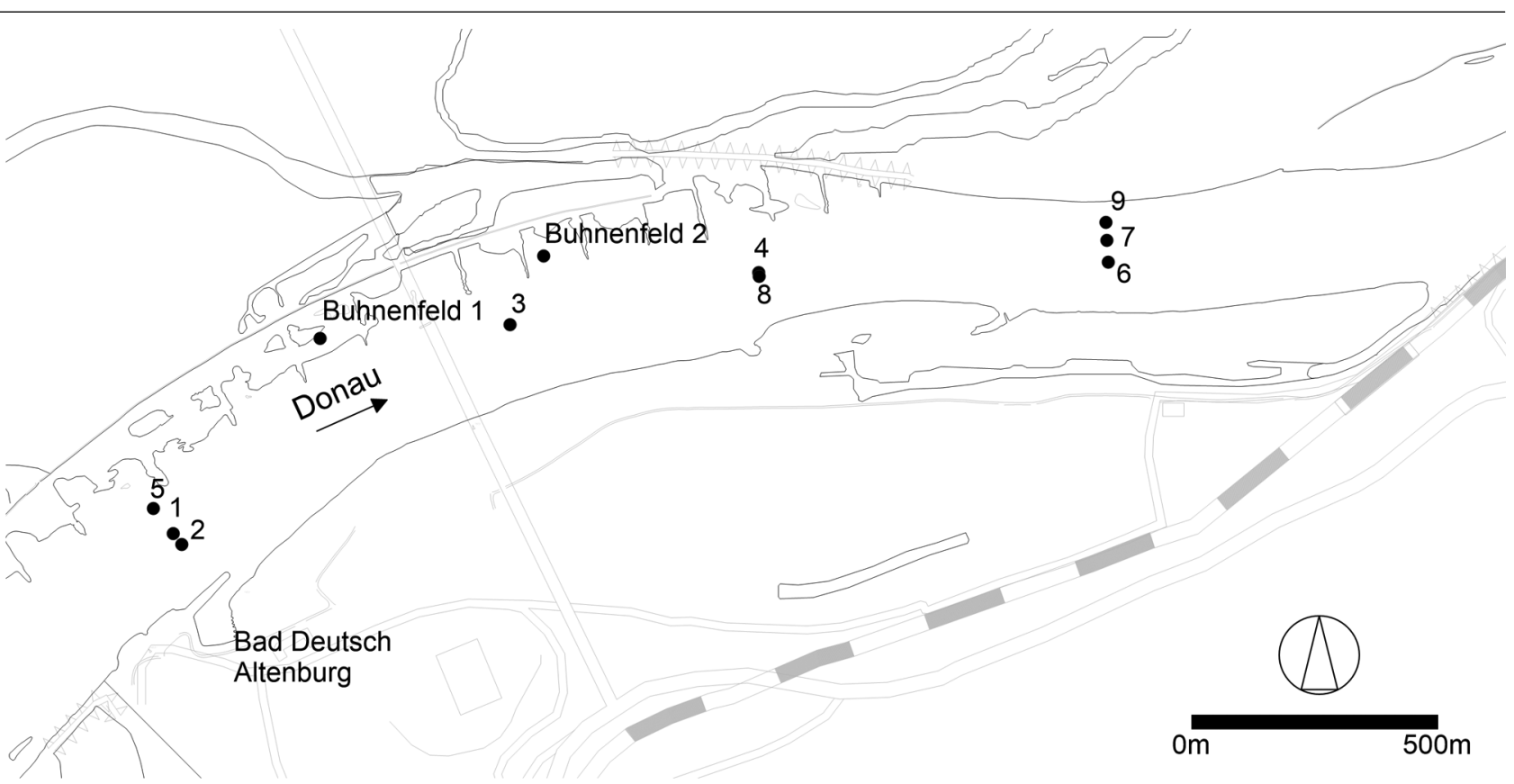

Abb. 2 Lageplan der Freeze-Panels aus den Jahren 2006 und 2015
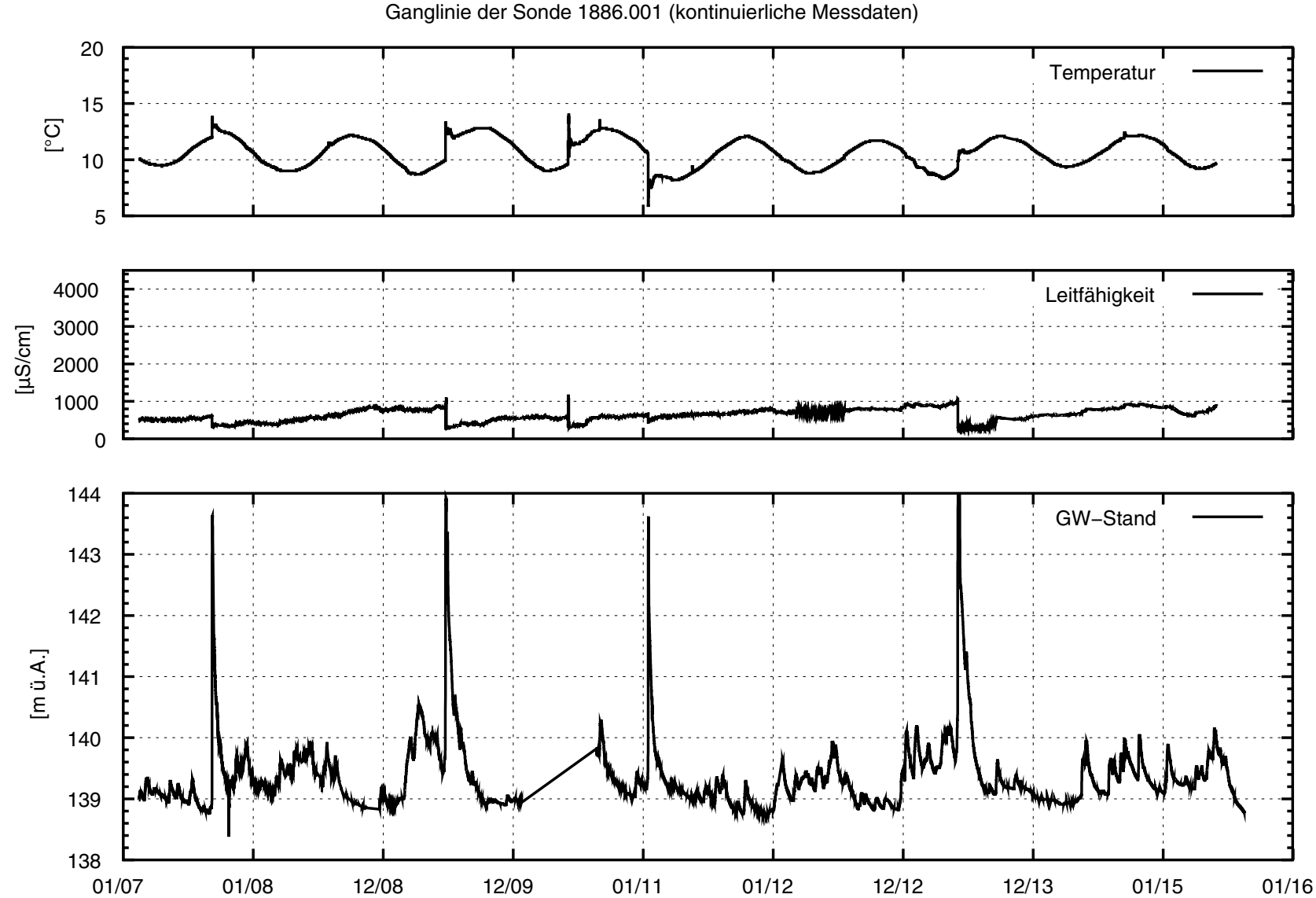

Abb. 3 Gemessene Zeitreihen beispielhaft für die Grundwassermessstelle 1886,001 (siehe Abb. 1 für den Lageplan) mit kontinuierlicher Datenerfassung von Grundwasserstand, -temperatur und Leitfähigkeit 


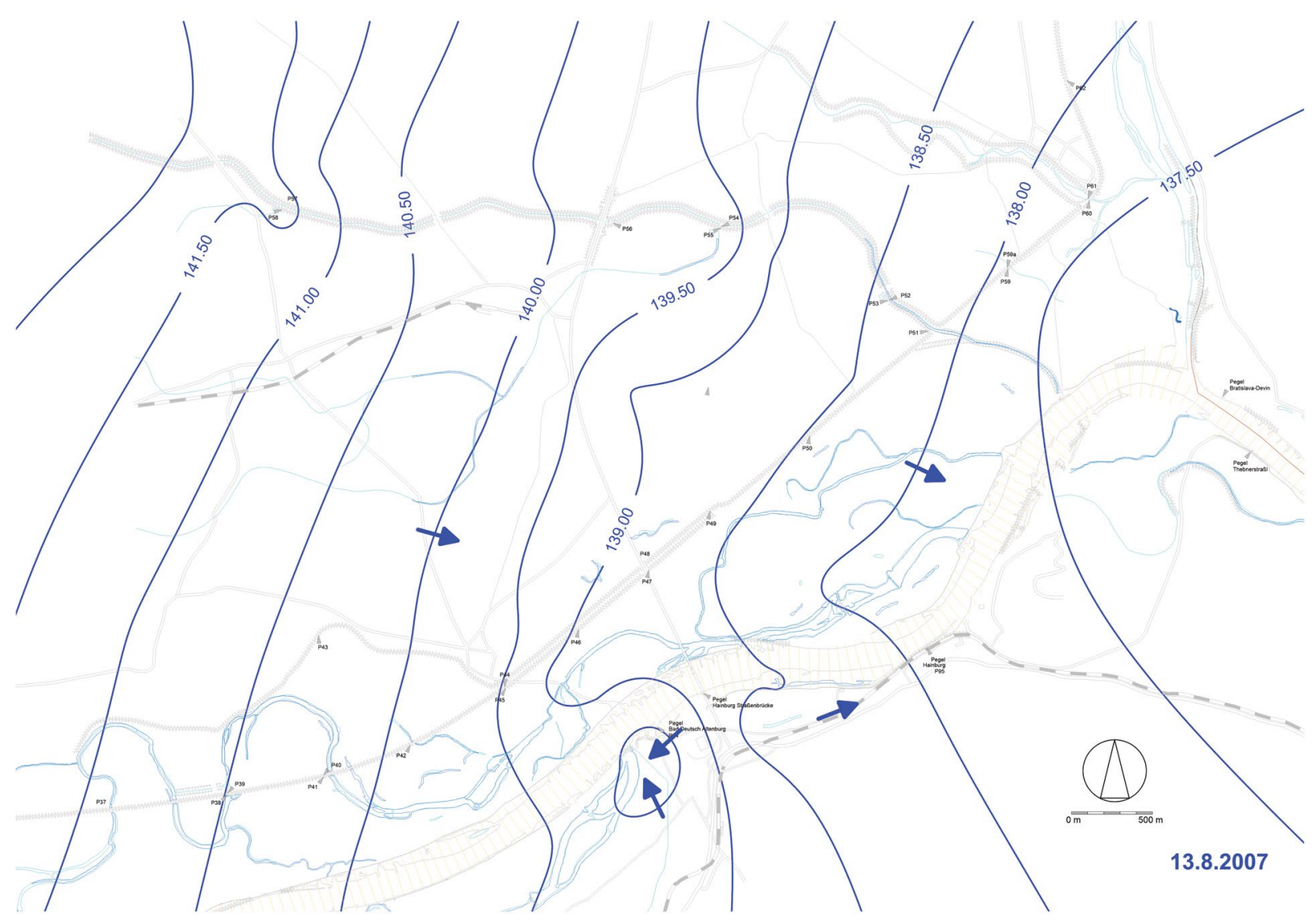

Abb. 4 Isolinien des Grundwasserstandes bei Mittelwasserführung der Donau vor Baumaßnahmen (13.8.2007)

lichen Terminwerten -linear interpoliert wurden. Neben den Grundwasserständen wurden dabei auch die Wasserspiegel der Donau mitberücksichtigt. Ausgehend von diesen Daten wurden die Grundwasserstände mithilfe des Kriging-Verfahrens mit einer räumlichen Diskretisierung von $50 \mathrm{~m}$ interpoliert.

\subsection{Untersuchungen der}

Gewässersohle entlang der Donau und im Johler Arm

Die „Freeze-Panel“-Technik (Blaschke et al. 2002) erlaubt durch Gefrieren mit flüssigem Stickstoff die Entnahme einer ungestörten Bodenprobe der oberen 20 bis $30 \mathrm{~cm}$ der Gewässersohle. Die noch gefrorene Bodenprobe wird in einem Sandbett auf einer Plastikfolie gelagert. Nach dem Auftauen wird zur Dokumentation der obersten Schicht ein Foto gemacht und anschließend die Probe je nach Struktur in meist zwei Schichten (Deckschicht und eine Unterschicht) für die Siebanalyse händisch geteilt. Die Sieblinienauswertung für jede der Schichten erfolgt nach ÖNORM B 4412. Es wurden insgesamt 11 FreezePanels vor Beginn der Bauphase im Jahr 2006 und 10 Freeze-Panels nach Bauende im Jahr 2015 im Naturversuch BDA Donau Strom-km 1887,5-1884,5 genommen (Abb. 2).

Die Dokumentation der Stromsohle im Hauptstrom der Donau mit Fotos erfolgte vor Umsetzung der Baumaßnahmen im Oktober 2006. Die Methodik ist ausführlich in Gutknecht et al. (1998) beschrieben. Es wurden alle $100 \mathrm{~m}$ zwischen dem Strom-km 1884,400 und 1887,200 Profile mit jeweils 5 bis 8 Standpunkten im Profil aufgenommen. In den Buhnenfeldern wurden die Aufnahmen im Oktober 2007 und im Johler Arm im Jänner 2008 gemacht. Die Aufnahmen nach den Baumaßnahmen fanden im November 2015 statt.

Die hydraulische Durchlässigkeit der Sohle des Johler Arms wurde mithilfe von Versickerungsversuchen untersucht. Dazu wurden an jeweils zwei repräsentativen Standorten entlang des Johler Arms vor den, während der und nach den Baumaßnahmen Standrohrversuche durchgeführt. Die Auswertung der Versickerungsversuche erfolgte nach ÖNORM B 4422-2. Da diese Methodik nach Anbindung des Johler Arms an die Donau aufgrund der nun vorhandenen Strömung nicht mehr technisch umsetzbar war, wurde für die Versuche im Jahr 2015 eine vereinfachte Methodik angewandt. Dazu wurde ein PVC-Rohr in etwa $50 \mathrm{~cm}$ Tiefe eingeschlagen und bis zum Anschlag mit Wasser befüllt. Danach wurde der fallende Wasserstand im Rohr mit Lichtlot gemessen und die hydraulische Leitfähigkeit aus der Differenz des Wasserstands pro Zeitintervall ausgewertet.

\subsection{Interaktion der Donau mit dem Grundwasser}

Die Interaktion von Grundwasser mit der Donau und den Seitenarmen der Donau wird mit einer Analyse der gemessenen 


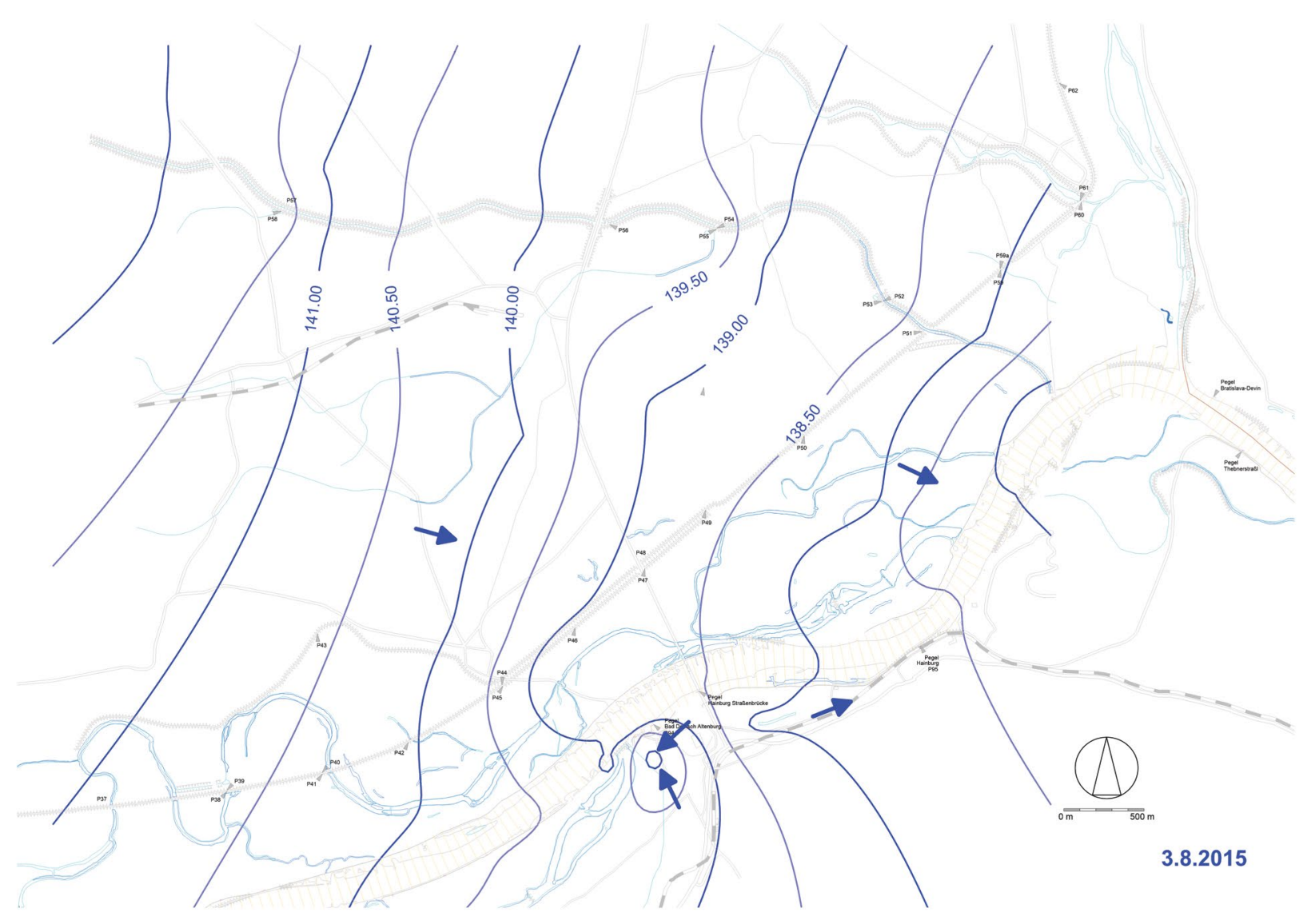

Abb. 5 Isolinien des Grundwasserstandes am 9.10.2015, nach den Baumaßnahmen, bei Mittelwasserführung der Donau und ähnlichen Grundwasserverhältnissen wie in Abb. 4

Wasserstandsganglinien in mehreren Messstellen untersucht. Aus den Höhenunterschieden der Grundwasserstände untereinander und zum Donauwasserstand beim Pegel Bad Deutsch-Altenburg (Abb. 1) ist zu erkennen, ob effluente (die Donau erhält Wasser aus dem Grundwasserkörper) oder influente Verhältnisse (die Donau infiltriert ins Grundwasser) vorherrschen. Die Donau ist aufgrund ihres Charakters als Gebirgsfluss und der zahlreichen stromaufwärts gelegenen Kraftwerksbetriebe einer starken zeitlichen Dynamik unterworfen (Blöschl et al. 2014; Merz et al. 2013). Die Grundwasserstände reagieren auf diese Dynamik mehr oder weniger stark, abhängig von der Distanz zum Ufer, der hydraulischen Durchlässigkeit und der Porosität des Grundwasserkörpers und des Grundwassergefälles (Derx et al. 2010). Die kontinuierlichen Messdaten auf Stundenbasis ermöglichen eine Darstellung dieser zeitlichen Dynamik der Grundwasserstände.

\subsection{Untersuchungen der Grundwasserqualität}

Für die Bestimmung der Grundwasserqualität wurden Proben von acht ausgewählten Messstellen analysiert, welche sich auf beiden Seiten der Donau befinden (Abb. 1). Die chemische Grundwasserqualität wurde an 3 Terminen vor Baubeginn und alle 2 Monate ab Baubeginn bis September 2015 untersucht. Zusätzlich wurde jeweils vor Baubeginn und nach Bauende nach einem DonauHochwasser $\left(<5000 \mathrm{~m}^{3} / \mathrm{s}\right)$ eine 14-tägige Probenahme über 3 Monate durchgeführt. Die mikrobiologische Grundwasserqualität wurde an jeweils drei Beprobungsterminen vor Baubeginn und ab 2 Monaten nach Bauende untersucht. Vor jeder Probenahme wurde das Grundwasser zunächst abgepumpt bis sich eine kontinuierliche elektrische Leitfähigkeit, pH-Wert und Temperatur einstellten. Die Probenahmen des Grundwassers erfolgten als Stichproben aus dem Pumpenschlauch einer Unterwasser- pumpe bzw. Oberwasserpumpe bei den Sonden mit geringem Durchmesser. Die Probenahmen der Donau erfolgten als Schöpfprobe vom linken Donau Ufer aus (Abb. 1). Die Auswertung der chemischen und mikrobiologischen Wasserqualität erfolgte im Labor für Nitrat und Sulfat nach ÖNORM EN ISO 10304-1, für gelösten Sauerstoff nach ÖNORM EN 25814, für gelösten organischen Kohlenstoff nach ÖNORM EN 1484, für E.coli nach ÖNORM EN ISO 9308-1 und für Enterokokken nach ÖNORM EN ISO 7899-2.

\section{Ergebnisse}

\subsection{Entwicklung der} Grundwasserspiegellagen

Bei der Entwicklung der Grundwasserspiegellagen zeigte sich ein gleich bleibender linearer Trend bei allen untersuchten Grundwassermessstellen bis Juni 2015, wie in Abb. 3 beispielhaft für die Messstelle 1886.001 dargestellt. 


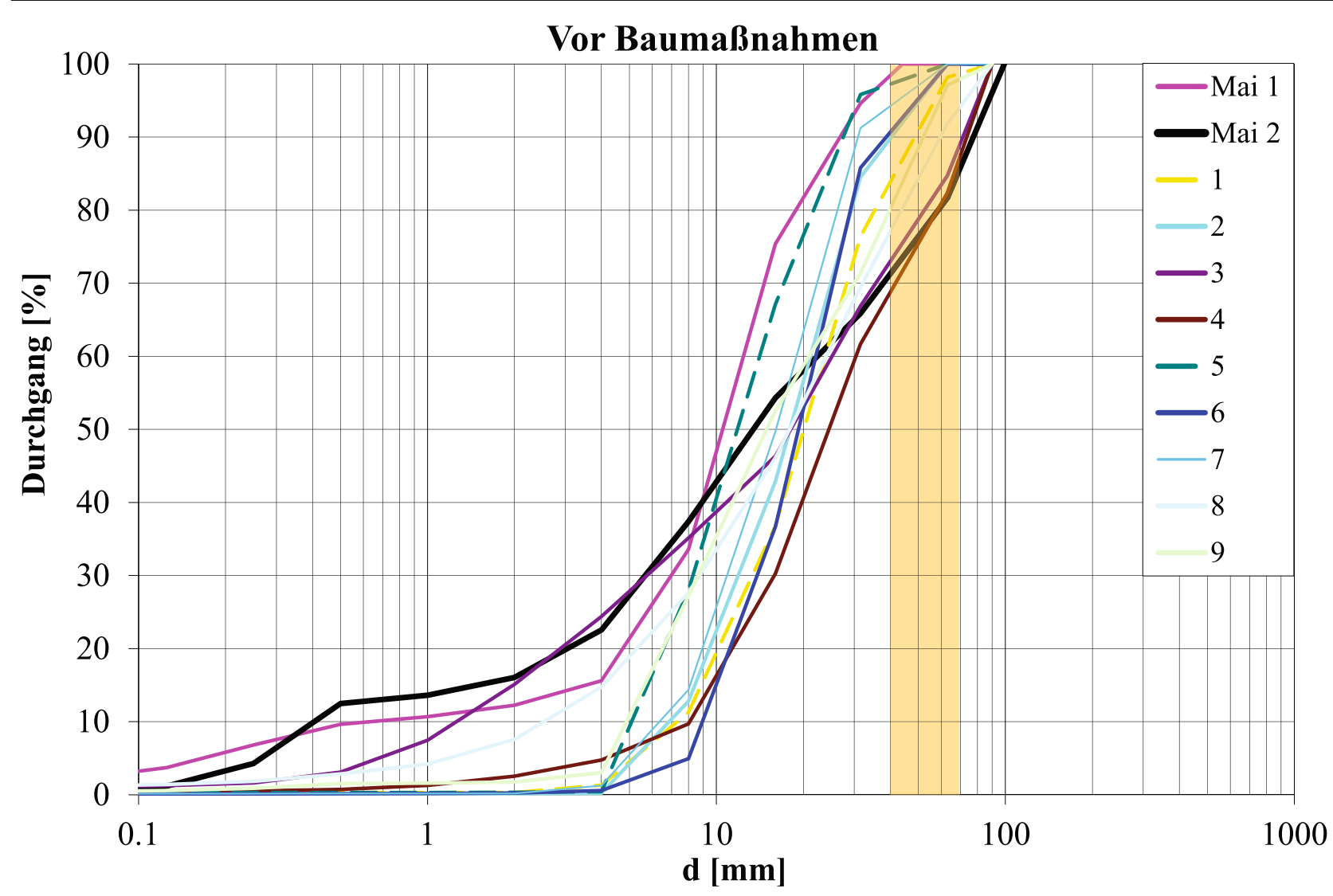

Abb. 6 Freeze-Panel-Auswertung vor den Baumaßnahmen im Jahr 2006 (Kornanteil 40/70 mm, StandardGSV ist gelb markiert)

Von Juni bis Dezember 2015 zeigte sich ein fallender Trend, da diese Periode von extremer Trockenheit bzw. wenig Niederschlag gekennzeichnet war. Die Grundwasserisolinien und -strömungsrichtungen zu ausgewählten Zeitpunkten vor und nach den Baumaßnahmen bei mittleren Donauwasserführungen sind in den Abb. 4 und 5 dargestellt. Es zeigt sich in beiden Fällen deutlich, dass im Allgemeinen Grundwasser vom Hinterland zur Donau strömt und exfiltriert.

\subsection{Untersuchungen der}

Gewässersohle entlang der Donau und im Johler Arm

Die Untersuchungen der Donausohle entlang des Hauptstroms und in zwei ausgewählten Buhnenfeldern mittels Siebanalysen und Fotodokumentation werden im Folgenden dargestellt. Im Anschluss daran werden die Ergebnisse der Versickerungsversuche gezeigt, in Form von hydraulischen Leitfähigkeiten der Gewässersohle des Johler Arms. Die Ergebnisse vor, während und nach den Baumaßnahmen werden einander gegenübergestellt.

Die Abb. 6 und 7 zeigen die Darstellung der Siebanalysen für die Freeze-Panels in der Pilotversuchsstrecke vor und nach den Baumaßnahmen. Der gelb markierte Bereich zeigt die Kornfraktion 40/70 mm, welche häufig bei der Zusammensetzung der GSV gewählt wird (Liedermann et al. 2016, in dieser Ausgabe). Beim Vergleich der Abbildungen erkennt man eine deutliche Vergröberung des Sediments in diesem gelb markierten Bereich. Auch in den Buhnenbereichen kommt es zu einer Vergröberung der Sedimente. Der Vergleich der Fotodokumentation der Stromsohle, hier beispielhaft für den Bereich Strom-km 1886,330 207 m bzw. 205 m vom linken Ufer der Donau dargestellt, zeigt ebenfalls eine deutliche Vergröberung der obersten Sedimentlagen (vergleiche Abb. 8 und 9).

Die Auswertungen der Versickerungsversuche im Johler Arm zeigen, dass sowohl vor Baubeginn im Jahr 2007 als auch während der Bauphase im Jahr 2013 und nach Bauende im Jahr 2015 kiesige Sohlverhältnisse durchmischt mit Fein- sedimentauflagen vorlagen (s. Abb. 10, beispielhaft für das Jahr 2013). Die Versuchsauswertung vor Baubeginn ergab für einen Standort im Einlaufbereich des Johler Arms (Aulehmboden) einen Wert für die hydraulische Durchlässigkeit ( $\mathrm{k}_{\mathrm{f}}$-Wert) von $2 \times 10^{-8} \mathrm{~m} / \mathrm{s}$, welcher einen extrem gering durchlässigen Boden charakterisiert. Die weiteren Versuche an weiteren typischen Standorten im Johler Arm erlaubten aufgrund der dort vorhandenen großen Durchlässigkeit keine Messung bzw. Auswertung. Es war an diesen Stellen mit kiesiger Oberfläche mit einem $\mathrm{k}_{\mathrm{f}}$-Wert von $<2 \times 10^{-2} \mathrm{~m} / \mathrm{s}$ zu rechnen. Diese extrem durchlässigen Kiesflächen wechselten sich mit dem sehr dichten Aulehmboden im Johler Arm ab (Derx und Blaschke 2015). Somit ergaben sich Bereiche, in denen bei einer Durchströmung des Johler Armes eine sehr rasche Versickerung von Donauwasser auftritt. In den Bereichen einer Feinsedimentauflage war eine kaum messbare Versickerung vorhanden. Die Versuchsauswertung während der Bauphase im Oktober 2013 ergab für alle drei gewählten Standorte (bei Wasseranschlagslinie, 


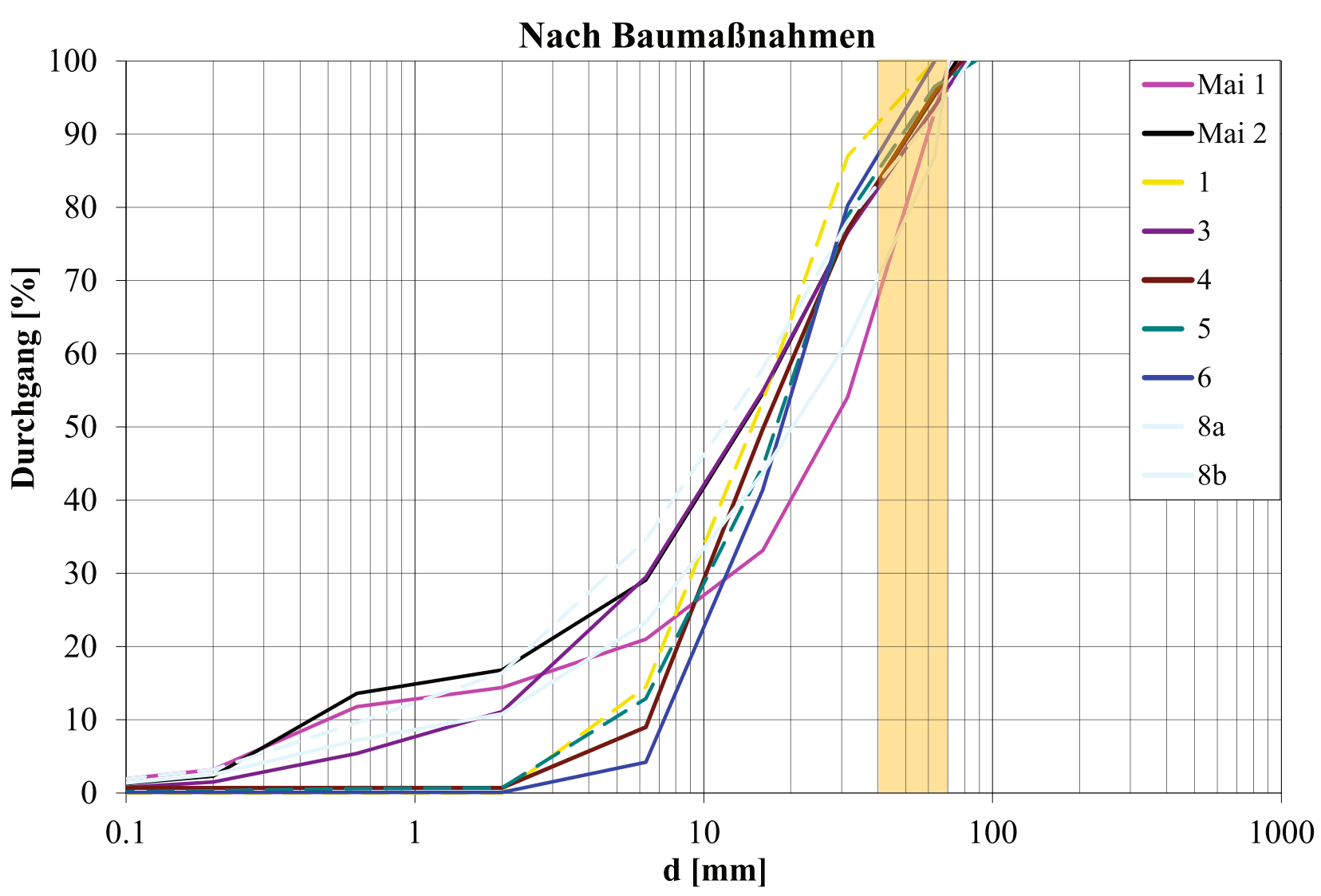

Abb. 7 Freeze-Panel-Auswertungen nach den Baumaßnahmen im Jahr 2015 (Kornanteil 40/70 mm, Standard-GSV ist gelb markiert)

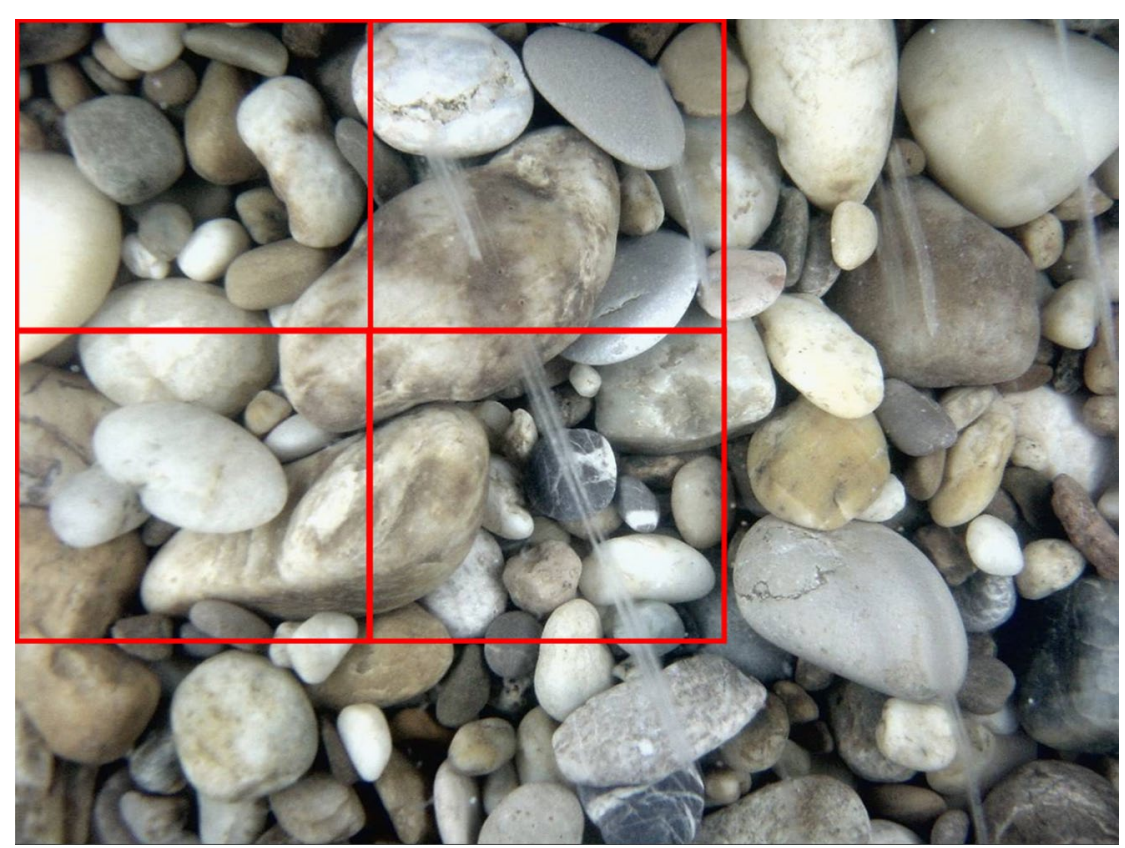

Abb. 8 Beispiel eines Fotos der Donausohle - Strom-km 1886,300, 207 m vom linken Ufer vor den Baumaßnahmen (roter Raster $5 \times 5 \mathrm{~cm}$ )
$1 \mathrm{~m}$ und $6 \mathrm{~m}$ über Wasseranschlagslinie) einen maximalen $\mathrm{k}_{\mathrm{f}}$-Wert von $5 \times 10^{-6} \mathrm{~m} / \mathrm{s}$. Die Bereiche im Johler Arm, bei denen vor Baubeginn hohe Durchlässigkeiten vorhanden waren, waren zu dem Zeitpunkt der erneuten Untersuchungen überströmt bzw. von den extrem gering durchlässigen Bodenschichten überlagert. Die Versuche nach Baudurchführung im Jahr 2015 im überströmten Bereich des Johler Arms ergaben Werte für die hydraulische Durchlässigkeit von $10^{-7}$ bis $10^{-5} \mathrm{~m} / \mathrm{s}$.

\subsection{Interaktion der Donau mit dem Grundwasser}

Die Analyse der Grundwasserganglinien vor und nach Beginn der Bauphase zeigt eine gleichbleibende Interaktion der Donau mit dem Grundwasser. Linksufrig im Marchfeld sind meist effluente Verhältnisse vorherrschend (Abb. 11). Dies gilt ebenso für die Messstelle 1883,015, wenn man die Höhendifferenz zum Pegel BDA mitberücksichtigt. Lediglich bei erhöhten Donauwasserführungen zeigt sich der Einfluss der Donau auf die Grundwasserstände und es kommt zur 


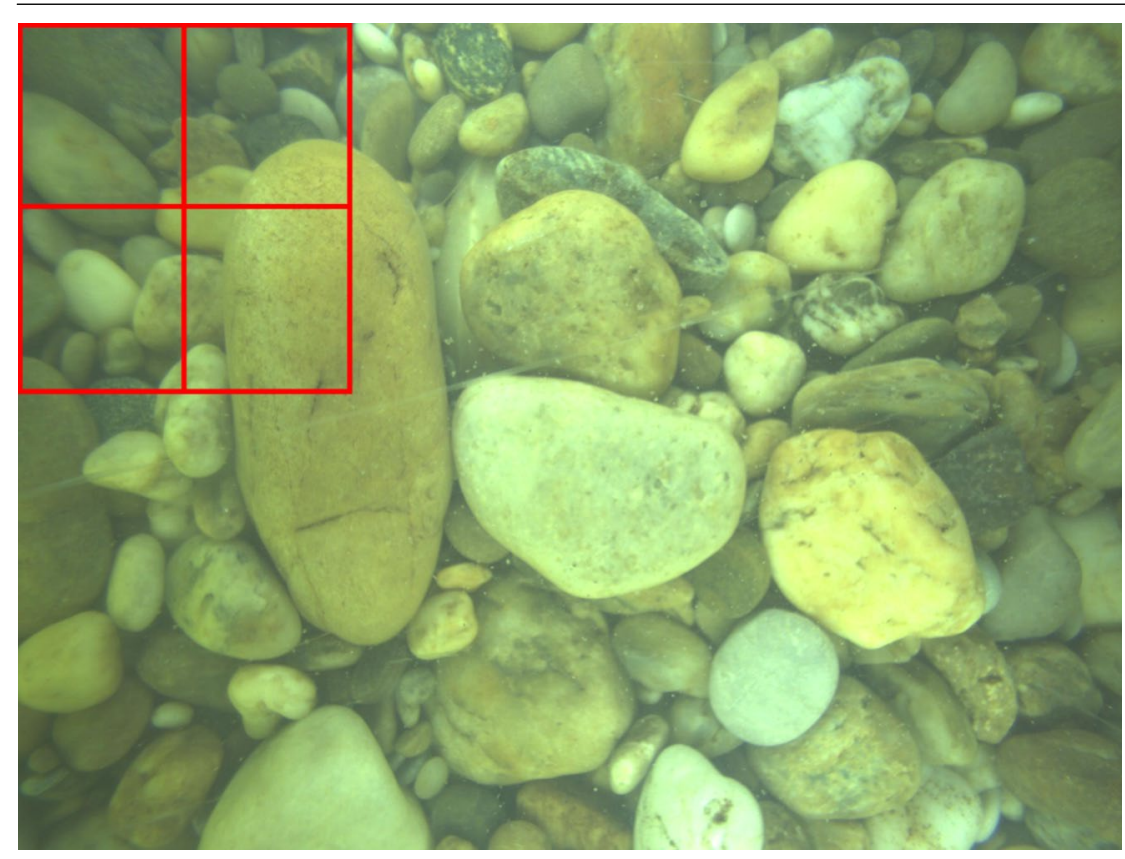

Abb. 9 Beispiel eines Fotos der Donausohle - Strom-km 1886,300, 205 m vom linken Ufer, 1 Jahr nach den Baumaßnahmen (roter Raster $5 \times 5 \mathrm{~cm}$ ) Der Bildausschnitt ist aufgrund einer technischen Änderung des Aufnahmegerätes größer als in Abb. 8
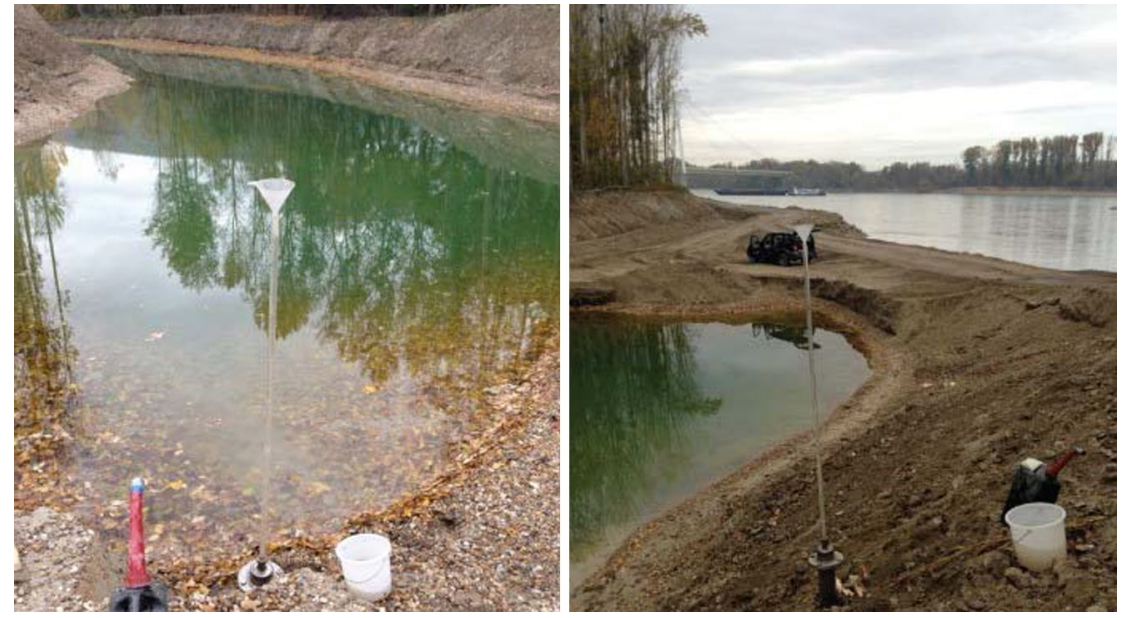

Abb. 10 Versickerungsversuche im Johler Arm im Jahr 2013

temporären Infiltration von Donauwasser in den Grundwasserkörper. Die Ganglien in Abb. 11 zeigen dies sehr anschaulich. Rechtsufrig wechseln sich effluente Verhältnisse im Bereich der Thermalbrunnen mit geringfügig influenten Verhältnissen im Bereich des Johler Arms ab.

Um die Entwicklung der Wasserstände im Johler Arm vor und nach den Baumaßnahmen zu untersuchen, wurden die berechneten Wasserspiegel zu charakteristischen Höhen im Johler Arm an der Stelle der Messstelle F6 (Abb. 1) keine Veränderung der Wasserspiegelhöhe im Johler Arm.

\subsection{Untersuchungen der Grundwasserqualität}

Die chemischen Analysen der Grundwasserqualität und der Donau für gelösten Sauerstoff $\left(\mathrm{O}_{2}\right)$, Nitrat $\left(\mathrm{NO}_{3}\right)$, gelösten organischen Kohlenstoffgehalt (DOC) und Sulfat $\left(\mathrm{SO}_{4}\right)$ sind in Abb. 13 dargestellt. Die Konzentrationen von gelöstem Sauerstoff, Nitrat und DOC im Grundwasser zeigen sich nach Baudurchführung erhöht. Ein möglicher Grund dafür könnte die Umlagerung der Feinsedimentauflagen im Altarmsystem während eines größeren Hochwasserereignisses wie etwa im Sommer 2013 sein. In diesem Milieu finden wichtige Abbauprozesse statt, welche mit einem Verbrauch an Sauerstoff der dort ansässigen Mikroorganismen einhergehen könnte (Bondar-Kunze et al. 2016, in dieser Ausgabe).

Die gemessenen Sulfatkonzentrationen zeigen vor und nach den Baumaßnahmen keine wesentliche Veränderung. Generell liegen die Werte in den von Thermalwasser beeinflussten Messstellen deutlich höher als in den sonstigen Messstellen, was durch die für Thermalwasser spezielle Charakteristik erklärbar ist (mit * markierte Messstellen in Abb. 13). Die mittlere Wassertemperatur und elektrische Leitfähigkeit in diesen Messstellen liegt ebenfalls deutlich höher, mit Werten von über $20^{\circ} \mathrm{C}$ bzw. über $3000 \mu \mathrm{S} / \mathrm{cm}$ (Abb. 14). Die mittleren Wassertemperaturen im Grundwasser zeigen keine wesentliche Veränderung vor und nach den Baumaßnahmen (Abb. 14). Die elektrischen Leitfähigkeiten im Grundwasser werden von unterschiedlichen Faktoren beeinflusst, wie z. B. von Niederschlägen und von Hochwässern. Während des Zeitabschnittes vor den Baumaßnahmen fand ein um rund $2 \mathrm{~m}$ höheres Hochwasser statt als während des Zeitabschnittes nach den Baumaßnahmen. Dadurch sind die geringeren mittleren Leitfähigkeiten nach den Baumaßnahmen bei einigen Messstellen auf der linken Donauseite (z. B. bei 1886.001) erklärbar (Abb. 14).

Abbildung 15 zeigt die Ergebnisse der mikrobiologischen Analysen. Die Auswertung enthält jeweils 3 Messtermine vor und nach den Baumaßnahmen. Diese Messreihen begannen jeweils 4 Wochen nach einem Hochwasser und wurden dann im Abstand von 2 Monaten durchgeführt. Die Fäkalindikatoren E.coli und 

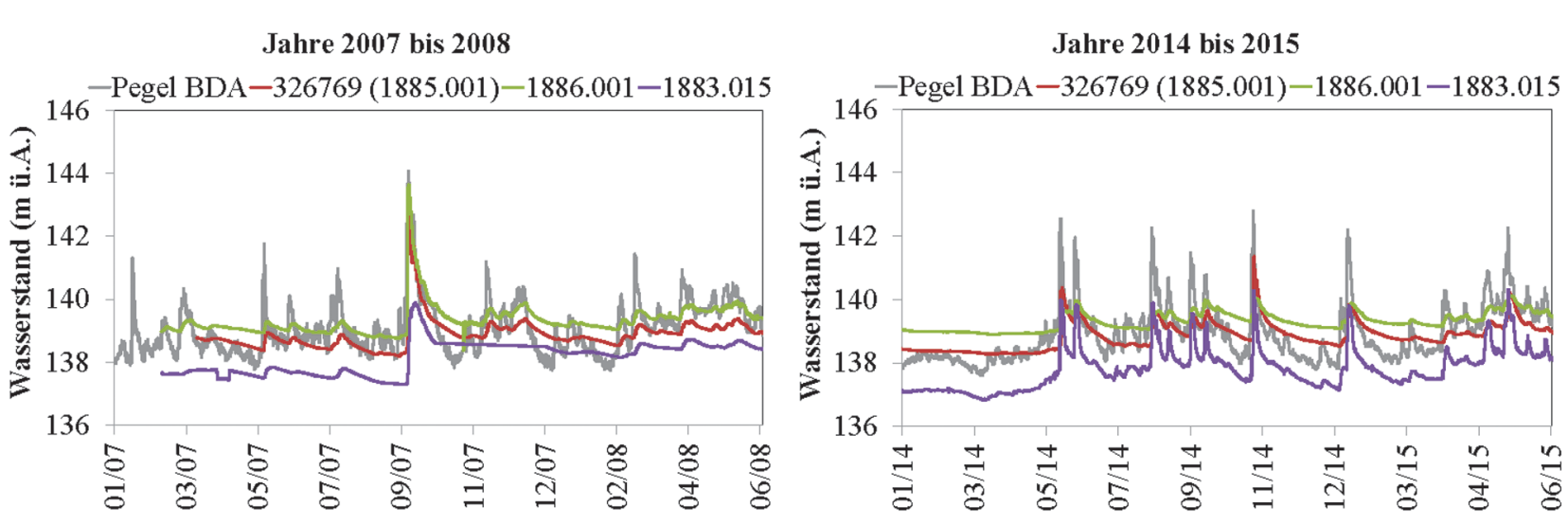

Jahre 2007 bis 2008
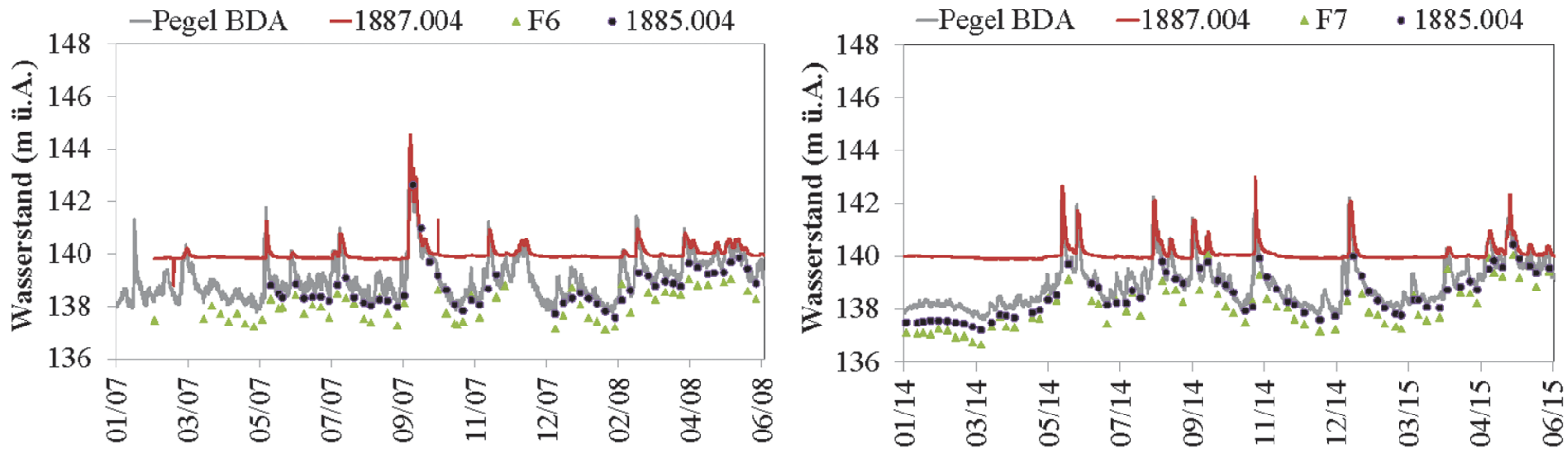

Abb. 11 Wasserstandsganglinien der Donau (Pegel Bad Deutsch-Altenburg) und von Grundwassersonden links- (oben) und rechtsufrig der Donau (unten); links vor den Baumaßnahmen, rechts danach

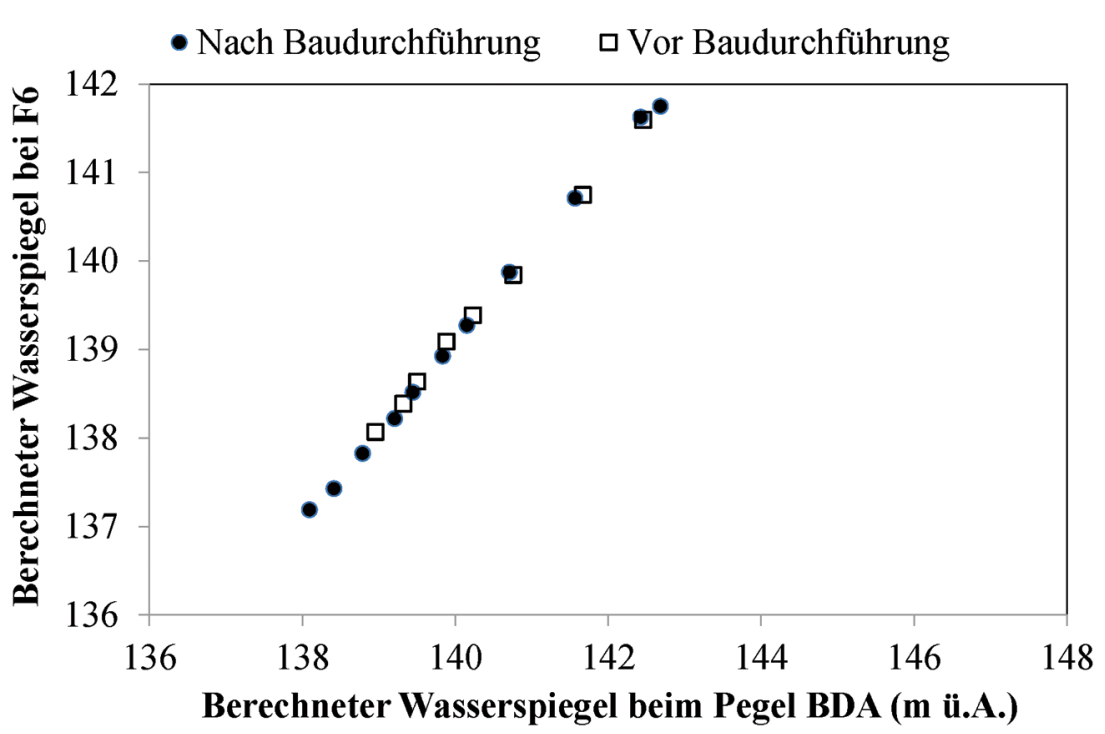

Abb. 12 Beziehung der berechneten Wasserspiegel der Donau (Pegel BDA) und beim Johler Arm (F6) vor und nach Baudurchführung zu verschiedenen Wasserständen (s. Liedermann et al. 2016, in dieser Ausgabe). Siehe Abb. 1 für die Lage der Messstellen
Enterokokken gelangen üblicherweise über kommunale Abwassereinleitungen in Flüsse (Mayer et al. 2015) und werden im Grundwasser durch die Filterwirkung des Bodens meist sehr rasch abgebaut. Im Vergleich zu E.coli können Enterokokken in der Regel länger in der Umwelt überleben, da sie gegenüber vielen Umweltfaktoren widerstandsfähiger sind. Aufgrund erhöhter Temperatur und Leitfähigkeiten, wie sie etwa unter den physikalisch-chemischen Bedingungen von Thermalwasser gegeben sind, kann diese unterschiedliche Überlebensfähigkeit (Persistenz) besonders ausgeprägt sein. Wie schon zuvor erwähnt sind einige der Grundwassermessstellen von Thermalwasser beeinflusst (1887.005, 1887.032, 1887.004, 1886.022). Die Ergebnisse für die von Thermalwasser beeinflussten Messstellen zeigen, dass der Fäkalindikator E.coli sowohl vor als auch nach den Baumaßnahmen kaum nachgewiesen werden konnte. Enterokokken konnten in höheren Konzentrationen in einigen dieser Messstellen nachgewiesen werden. Diese im Vergleich zu E.coli er- 

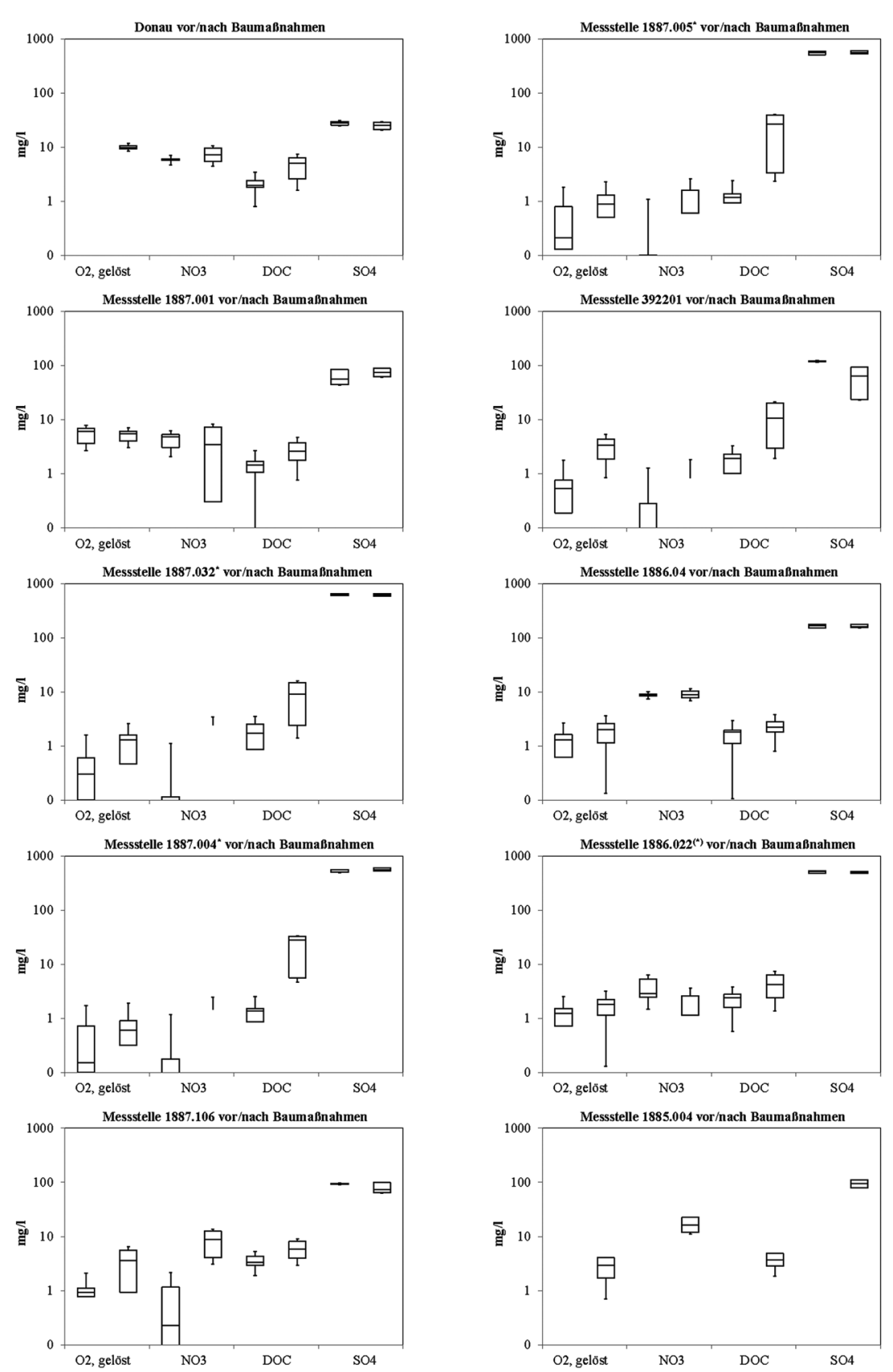

Abb. 13 Ergebnisse der chemischen Analysen für gelösten Sauerstoff, Nitrat, DOC und Sulfat vor bzw. nach Baumaßnahmen bis Dezember 2015. * bezeichnet von Thermalwasser beeinflusste Messstellen

höhten Konzentrationen könnten, wie bereits oben erwähnt, durch ein längeres Überleben in der Umwelt erklärbar sein. Darüber hinaus können diese erhöhten Konzentrationen auch durch Vermehrung und Wachstum bedingt sein. Vermehrung unter günstigen Wachstumsbedingungen, wie etwa unter gegebenen Thermalwasserbedingungen, erscheint durchaus möglich. Der Nachweis dieser Fäkalbakterien kann daher nicht als eintieferen Grundwasserhorizont ist anzunehmen, dass dieser mit dem Grundwasserkörper auf der rechten Donauseite verbunden ist. Die folgenden Messstellen waren wenig bis nicht von Thermalwässern beeinflusst: 1887.001, 1887.003, 1886.04, 1887.106. Bei denjenigen Messstellen, welche sich in unmittelbarer Ufernähe der Donau befinden (1887.001, 1886.04, 1886.022), zeigten sich bei der Messreihe, welche nach den Baumaßnahmen durchgeführt wurde, um etwa 1 $\log _{10}$-Stufe geringere Konzentrationen als zuvor (Abb. 15).

\section{Zusammenfassung}

Die bisherigen Analysen der Grundwasserverhältnisse vor und nach den Baumaßnahmen im Pilotversuch Bad Deutsch-Altenburg zeigten keine wesentliche Beeinflussung des Grundwassers durch die Baumaßnahmen. Dies wurde durch den Vergleich der Grundwasserstandshöhen und -strömungsrichtungen und der chemischen und mikrobiologischen Wasserqualität im Grundwasser und in der Donau vor und nach den Baumaßnahmen dargestellt. Die Interaktion des Grundwassers mit der Donau blieb ebenso intakt.

Die Analyse der ungestörten Proben der Donausohle mithilfe der FreezePanel-Technik sowie die Dokumentation der Stromsohle mit Fotos zeigen eine deutliche Vergröberung des Sediments entlang des Hauptstroms und in den Buhnenbereichen. Dies deutet auf das Vorhandensein der eingebrachten GSV hin.

Die Analysen der Grundwasserqualität zeigen wie zu erwarten den Einfluss von Thermalwässern im Bereich von Bad Deutsch-Altenburg.

Der geringe Einfluss der Baumaßnahmen auf die vorherrschenden quantitativen und qualitativen Grundwasserverhältnisse im Bereich des Pilotversuchs Bad Deutsch-Altenburg ist weitgehend auf die effluenten Verhältnisse zwischen Grund- und Oberflächenwasser zurückzuführen. Eine Übertragung dieser Ergebnisse auf andere Streckenabschnitte der Donau mit anderen Rahmenbedingungen ist daher nur bedingt möglich. Die Ergebnisse weisen deutlich darauf hin, dass eine Aufrechterhaltung der Interaktion des Grundwassers mit der Donau und deren Seitenarme eine wesentliche Bedeutung für die Erhaltung der Grundwasserverhältnisse haben. Deshalb werden weitere Maßnahmen gegen ein Absinken der Oberflächenwasserstände erforderlich sein. 


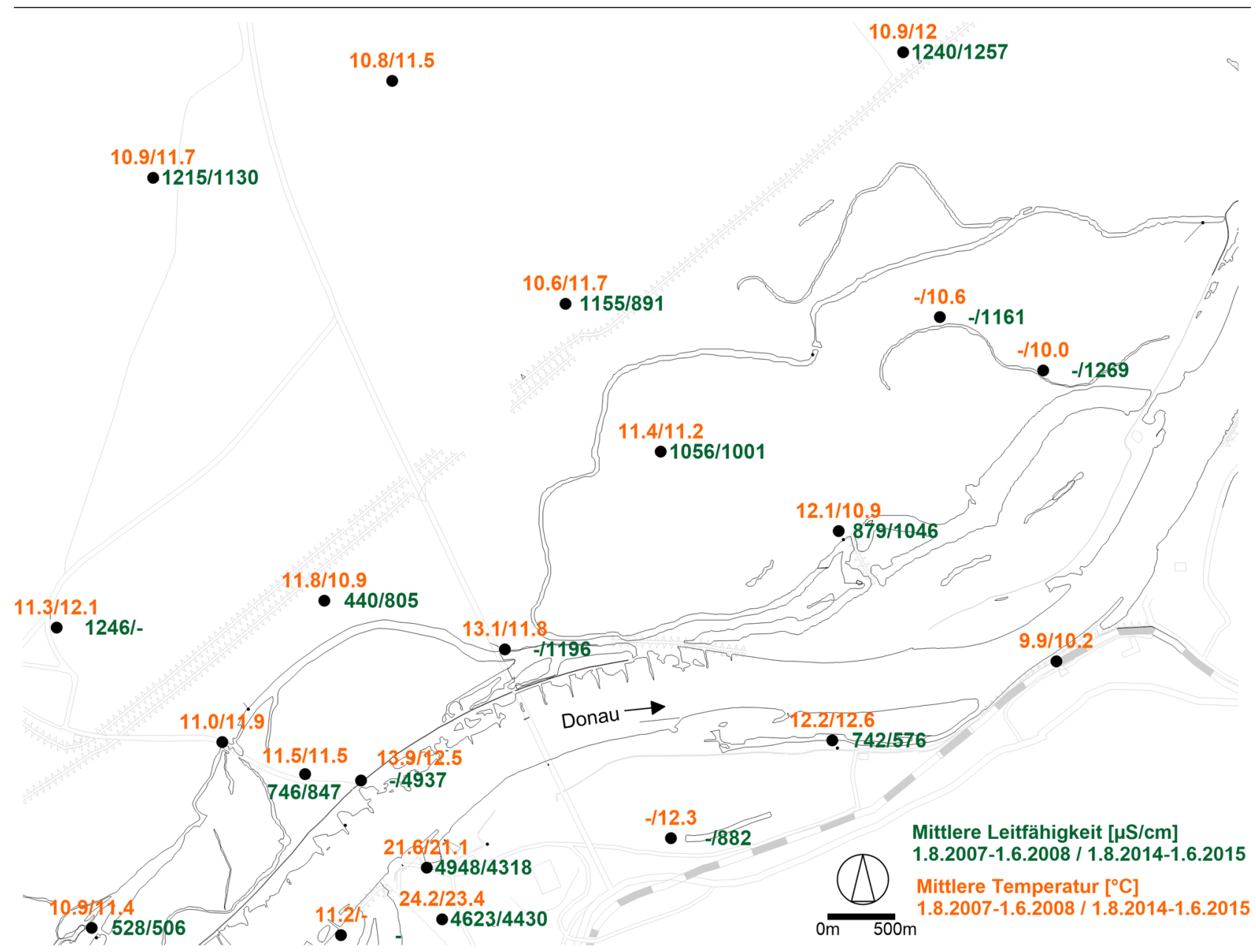

Abb. 14 Gemessene mittlere Wassertemperaturen und elektrische Leitfähigkeiten in den Grundwassermessstellen und in der Donau bei Pegel Hainburg vor/nach den Baumaßnahmen

\section{Danksagung}

Wir möchten der viadonau - Österreichische Wasserstraßen $\mathrm{GmbH}$ für die Unterstützung bei den über die Behördenauflagen hinausgehenden Messungen, die $\mathrm{zu}$ diesen Analysen wesentlich beigetragen haben, danken. Weiters möchten wir Hubert Keckeis, Reinhard Krusch, Philipp Reiner, Michael Exner-
Kittridge und Peter Haas für die Unterstützung bei der Fotodokumentation der Sohle danken.

\section{Einhaltung ethischer Richtlinien} Attribution License. Dadurch sind die Nutzung, Verteilung und Reproduktion erlaubt, sofern der/die Originalautor/en und die Quelle angegeben sind.

Interessenkonflikt Es besteht kein Interessenkonflikt. 

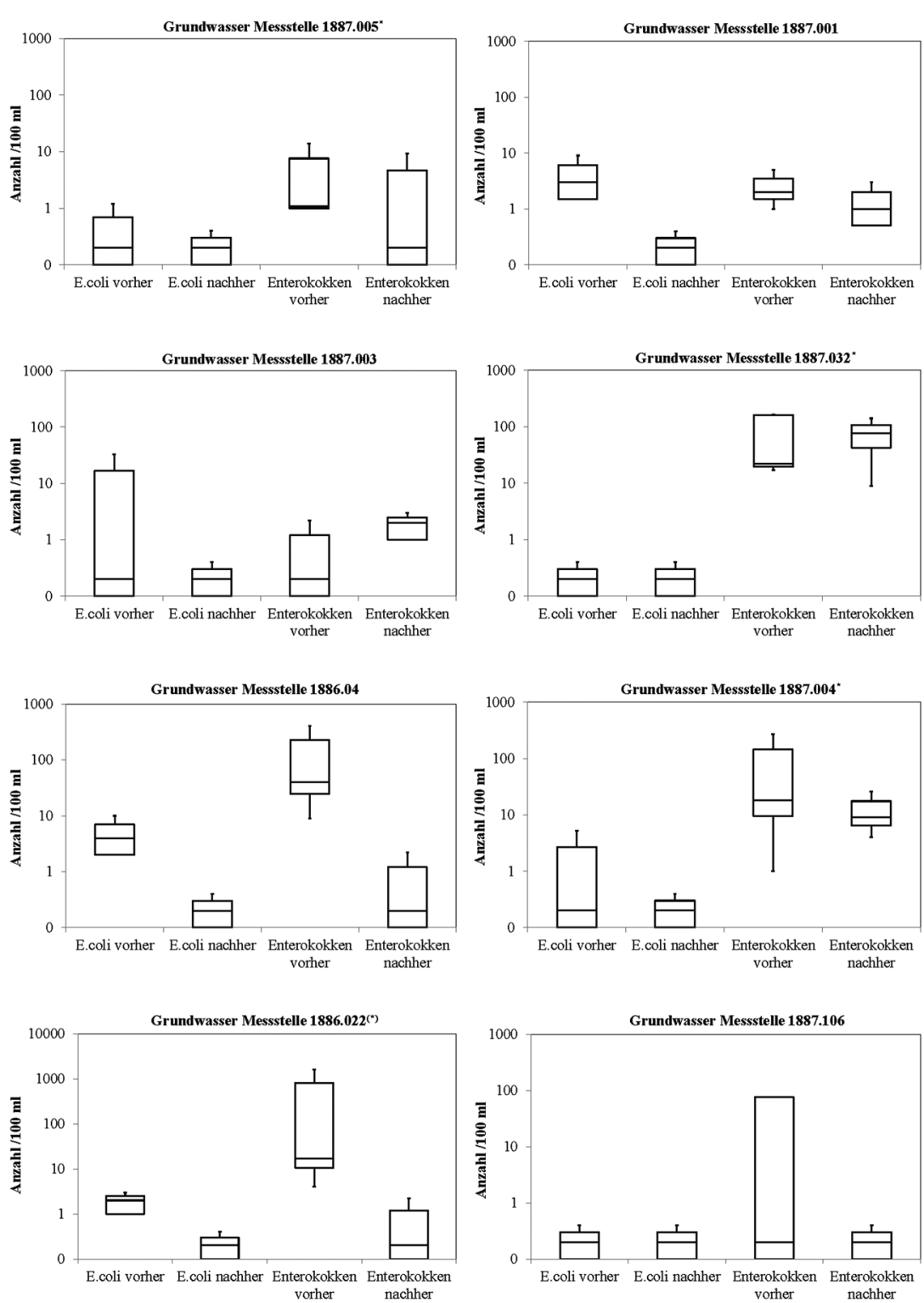

Abb. 15 Ergebnisse der mikrobiologischen Analysen vor und nach den Baumaßnahmen bei 8 Grundwassermessstellen (siehe Abb. 1, Lageplan). * bezeichnet von Thermalwasser beeinflusste Messstellen 


\section{Literatur}

Blaschke, A.P., Braunshofer, R., Feregyhazy, H., Gutknecht, D., Habersack, H., Karner, Ch., Mayr, P., Schmalfuß, R., Schober, St., Sengschmitt, D., Steiner, K.-H. (2002): Kolmationsprozesse am Beispiel des Stauraumes Freudenau, Projektphase II - Vollstau. Forschung im Verbund, Schriftenreihe Band 75; Verbund, Eigenverlag, Wien.

Blaschke, A.P., Derx, J. (2009): Naturversuch Bad Deutsch-Altenburg - Strom km 1887,5-1884,5. Beweissicherungsbericht 2007 - Messprogramm 2005-2009 Grundwasser. viadonau - Österreichische Wasserstraßen Gesellschaft mbH.

Blöschl, G., Nester, T., Parajka, J., Komma, J. (2014): Hochwasserprognosen an der österreichischen Donau und Datenassimiliation. Hydrologie und Wasserbewirtschaftung 58, 64-72. Bondar-Kunze, E., Hein, T. (2016): Grundlagenerkenntnisse aus den Untersuchungen. Österreichische Wasser- und Abfallwirtschaft $68, \mathrm{H}$. $1-2, \mathrm{XX}-\mathrm{XX}$.

Derx, J., Blaschke, A. P., Blöschl, G. (2010): Three dimensional flow patterns at the stream-aquifer interface - a case study at the Danube. Advances in Water Resources 33(11), 1375-1387.

Derx, J., Blaschke, A.P. (2015): Analyse de Grundwasserverhältnisse im Pilotprojekt Bad Deutsch-Altenburg. Vortrag: Fachtagung Pilotprojekt Bad Deutsch-Altenburg, 16. Oktober 2015, Wien; In: Fachtagung Pilotprojekt Bad DeutschAltenburg - Erkenntnisse für Schifffahrt, Ökologie und Flussbau (2015), S. 59-62
Gutknecht, D., Blaschke, A., Schmalfuss, R., Sengschmitt, D., Steiner, K., Reichl, G. Feregyhazy, H., Herndl, G., Battin, T., Dreher, J. (1998): Kolmationsprozesse am Beispiel des Stauraumes Freudenau. Forschung im Verbund, Schriftenreihe Band 44, Eigenverlag, Wien.

Liedermann, M. G. et al. (2016): Erkenntnisse bezüglich Maßnahmen. Österreichische Wasserund Abfallwirtschaft 68, H. 1-2, XX-XX

Mayer, R.E., Vierheilig, J., Egle, L., Reischer, G.H., Saracevic, E., Mach, R.L., Kirschner, A.K.T., Zessner, M., Sommer, R., Farnleitner, A.H (2015): Automated sampling procedures supported by high persistence of bacterial fecal indicators and Bacteroidetes genetic microbial source tracking markers in municipal wastewater during short-term storage at $5^{\circ} \mathrm{C}$. Appl. Environ. Microbiol. 81 (15):5134-43.

Merz, R., Parajka, J., Nester, T., Blöschl, G. (2013): Zur raum-zeitlichen Dynamik des Laufwasserkraftpotentials in Österreich (On the space-time dynamics of the run-of-river hydropower potential in Austria) Elektrotechnik und Informationstechnik 130, 2-8.

ONORM EN 25814 (1.6.1993): Wasserbeschaffenheit - Bestimmung des gelösten Sauerstoffes, elektrochemisches Verfahren (ISO 5814:1990)
ÖNORM EN ISO 10304-1 (1.6.2012): Wasserbeschaffenheit - Bestimmung von gelösten Anionen mittels FlüssigkeitsIonenchromatographie - Teil 1: Bestimmung von Bromid, Chlorid, Fluorid, Nitrat, Nitrit, Phosphat und Sulfat

ÖNORM EN ISO 7899-2 (1.11.2014): Wasserbeschaffenheit - Nachweis und Zählung von intestinalen Enterokokken - Teil 2: Membranfiltrationsverfahren (ISO 7899-2:2000)

ONORM EN 1484 (1.8.1997): Wasseranalytik - Anleitungen zur Bestimmung des gesamten organischen Kohlenstoffs (TOC) und des gelösten organischen Kohlenstoffs (DOC)

ÖNORM EN ISO 9308-1 (1.12.2014): Wasserbeschaffenheit - Nachweis und Zählung von Escherichia coli und coliformen Bakterien - Teil 1: Membranfiltrationsverfahren für Wässer mit niedriger Begleitflora

ÖNORM B 4422-2 (1.6.2002): Erd- und Grundbau - Untersuchung von Böden - Bestimmung de Wasserdurchlässigkeit - Feldmethoden für oberflächennahe Schichten

ÖNORM B 4412 (1.7.1974): Erd- und Grundbau -

Untersuchung von Böden - Korngrößenverteilung 